\title{
Is CGCS 5926 a symbiotic X-ray binary?^
}

\author{
N. Masetti ${ }^{1}$, U. Munari ${ }^{2}$, A. A. Henden ${ }^{3}$, K. L. Page ${ }^{4}$, J. P. Osborne ${ }^{4}$, and S. Starrfield ${ }^{5}$ \\ 1 INAF - Istituto di Astrofisica Spaziale e Fisica Cosmica di Bologna, via Gobetti 101, 40129 Bologna, Italy \\ e-mail: masetti@iasfbo.inaf.it \\ 2 INAF - Osservatorio Astronomico di Padova, Sede di Asiago, via dell'Osservatorio 8, 36012 Asiago, Italy \\ 3 AAVSO, 49 Bay State Road, Cambridge, MA 02138, USA \\ ${ }^{4}$ Department of Physics \& Astronomy, University of Leicester, Leicester, LE1 7RH, UK \\ 5 School of Earth and Space Exploration, Arizona State University, Tempe, AZ 85287-1404, USA
}

Received 15 May 2011 / Accepted 12 September 2011

\begin{abstract}
We report on multiwavelength (X-ray to optical) follow-up observations of the carbon star CGCS 5926. These were motivated by the fact that this star is positionally coincident with a faint X-ray emitting object belonging to the ROSAT catalog of sources, thus suggesting a possible symbiotic X-ray binary (SyXB) nature for it. Our spectrophotometric optical data confirm the giant carbon star nature of the object and allow us to classify its spectral type as $\mathrm{C}(6,2)$. This classification places CGCS 5926 at a distance of $\sim 5 \mathrm{kpc}$ from Earth. $B V R_{\mathrm{C}} I_{\mathrm{C}}$ photometry of the star shows that it displays a variability of $\sim 0.3 \mathrm{mag}$ on timescales of months, with the star getting bluer when its brightness increases. Our photometric data indicate a periodicity of 151 days, which we explain as due to radial pulsations of CGCS 5926 on the basis of its global characteristics. The source is not detected at X-rays with Swift/XRT down to a $0.3-10 \mathrm{keV}$ band luminosity of $\approx 3 \times 10^{32} \mathrm{erg} \mathrm{s}^{-1}$. This nondetection is apparently in contrast with the ROSAT data; however we show that, even if the probability that CGCS 5926 can be a SyXB appears quite low, the present information does not completely rule out such a possibility, while it makes other interpretations even more unlikely if we assume that the ROSAT detection was real. This issue might thus be settled by future, more sensitive, observations at high energies.
\end{abstract}

Key words. stars: AGB and post-AGB - techniques: spectroscopic - stars: carbon - stars: individual: CGCS 5926

\section{Introduction}

Low mass X-ray binaries (LMXBs) are interacting systems composed of a compact object, neutron star (NS) or black hole, which is accreting from a late-type companion star, with mass generally $\lesssim 1 M_{\odot}$ and still on the main sequence (or possibly slightly evolved).

There exists however a handful of cases in which the donor star is actually a red giant: these, by analogy with the symbiotic binaries which are formed by an evolved late-type star and a white dwarf, are dubbed symbiotic X-ray binaries (SyXBs; see e.g. Masetti et al. 2006a).

Observationally, these systems are characterized by appreciable X-ray emission $\left(\sim 10^{32}-10^{34} \mathrm{erg} \mathrm{s}^{-1}\right.$; see Masetti et al. 2007, and references therein) positionally associated with a red giant star which spectroscopically does not show any abnormal features, with the possible exception of a continuum excess in the blue and ultraviolet ranges. A notable outlier is the source GX 1+4, which emits up to $\sim 10^{37} \mathrm{erg} \mathrm{s}^{-1}$ and shows several emission lines in the optical spectrum of the red giant companion (Chakrabarty \& Roche 1997; Munari \& Zwitter 2002). This is most likely due to the fact that, in this latter case, accretion onto the compact object takes place via Roche lobe overflow rather than via a stellar wind (but see Hinkle et al. 2006, for a different scenario). This allows the creation of a disk around the

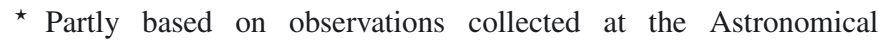
Observatories of Asiago and Loiano (Italy). accretor and makes the mass transfer phenomenon more efficient in terms of production and reprocessing of X-ray emission.

Moreover, X-ray pulsations, with periods ranging from hundreds up to tens of thousands of seconds, were detected from these systems: this indicates that the accreting compact object is a slowly rotating neutron star, the most extreme case being 4U 1954+319 ( $P_{\text {spin }} \sim 18400$ s; Corbet et al. 2008).

All the above characteristics make these systems rather unusual and, indeed, they are quite rare when compared to the number of known LMXBs (about 190, according to Liu et al. 2007): up to now, only 6 systems are firmly included in this subclass of LMXBs (see Masetti et al. 2007; Nespoli et al. 2010, and references therein) through coincidence between optical and $\mathrm{X}$-ray positions and subsequent confirmation via optical or nearinfrared (NIR) spectroscopy. Three more cases however exist, having either the confirmation still pending due to the lack of optical or NIR spectroscopy (1RXS J180431.1-273932: Nucita et al. 2007; 2XMM J174016.0-290337: Farrell et al. 2010), or with a still debated nature (IGR J16393-4643: see Nespoli et al. 2010; but also Thompson et al. 2006; and Corbet et al. 2010 , for a different source classification as a supergiant highmass X-ray binary).

Therefore, given the small number of known SyXBs, each new possible member of this subclass of LMXBs should be the object of an in-depth multiwavelength study to expand the sample. 


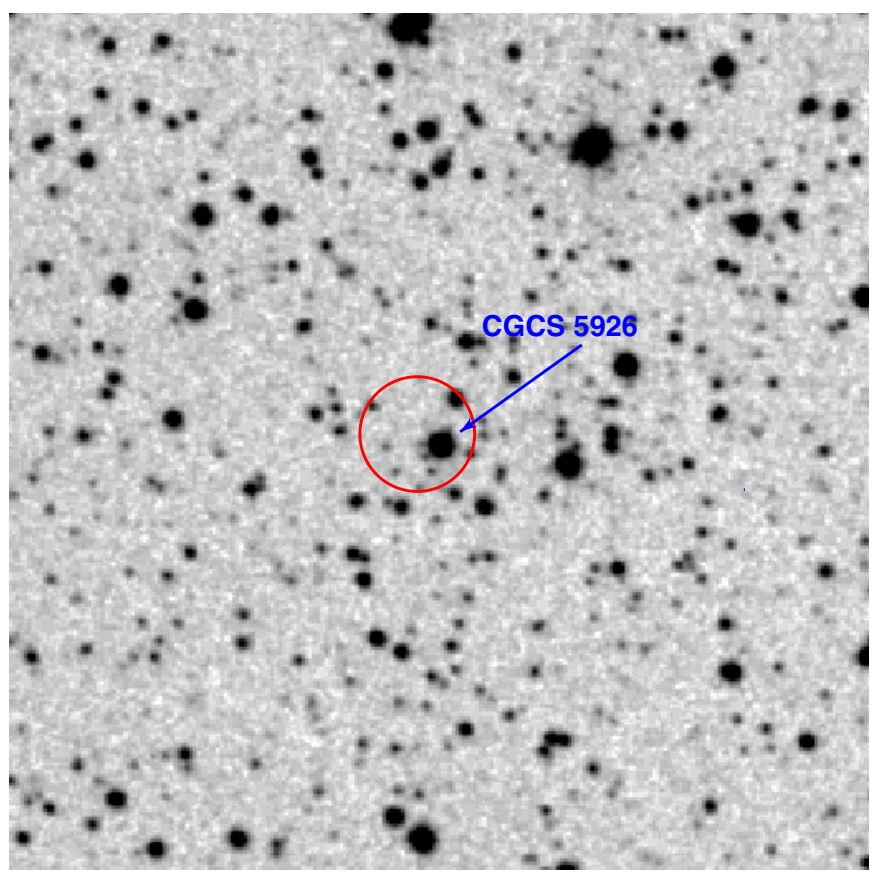

Fig. 1. DSS-II-Red image of the field of CGCS 5926 (the star is indicated by the arrow) with superimposed the 20" -radius $0.1-2.4 \mathrm{keV}$ ROSAT X-ray error circle. In the figure, North is at top and East is to the left. The field size is $5^{\prime} \times 5^{\prime}$.

We thus focused our attention on star CGCS 5926, which was classified by Maehara \& Soyano (1987) as a $V=14.8 \mathrm{mag}$ carbon star (thus a late-type giant) in the Cassiopeia region; according to the 2MASS catalog (Skrutskie et al. 2006), this object has NIR magnitudes $J=8.824 \pm 0.023, H=7.490 \pm 0.042$ and $K_{\mathrm{s}}=6.942 \pm 0.024$. Our interest in this star was drawn by the fact that (see Fig. 1) it is positionally inside the $20^{\prime \prime}$ error circle of the soft X-ray source 1RXS J234545.9+625256 belonging to the ROSAT All-Sky Survey Faint Source Catalog (Voges et al. 2000), which makes CGCS 5926 a SyXB candidate due to its optical spectral classification and its possible X-ray emission.

However, the lack of further information at optical, X-ray and other wavelengths does not make a secure case for inclusion of this star in the SyXB subclass. It is therefore worthy of further analysis by means of a specific spectrophotometric campaign in the optical range, as well as of a pointed observation with the use of an X-ray satellite affording localizations with a precision better than a few arcseconds.

We here report the results of optical, ultraviolet and X-ray observations of CGCS 5926 and a discussion of them, and we conclude that this star is either an X-ray variable or eclipsing SyXB, or that the low-significance ROSAT detection was spurious.

The outline of the paper is as follows: in Sect. 2 we describe our optical and X-ray observations, while Sect. 3 reports the results and Sect. 4 a discussion of them. Finally, in Sect. 5 we present the conclusions of our multiwavelength investigation of star CGCS 5926.

\section{Observational data}

\subsection{Optical}

Optical spectroscopy of CGCS 5926 was acquired on 14 October 2009 with the 1.82-m "Copernicus" telescope of the Asiago Astronomical Observatory (Italy) plus the AFOSC instrument (which carries a $1024 \times 1024$ pixel Tektronix TK1024 CCD). Using the Grism \#4 and a 1'.26 slit, this setup provided a dispersion of $4.2 \AA /$ pixel and a nominal coverage between 3500 and $7800 \AA$. The total exposure time was $2 \times$ 20 min centered at 02:03 UT. Spectroscopic observations to further explore the blue part of the optical range were secured at the Astronomical Observatory of Bologna in Loiano (Italy) with the 1.52-m "Cassini" telescope plus the BFOSC instrument carrying an EEV $1300 \times 1340$ pixel CCD: with the use of Grism \#6 and a slit width of $2^{\prime \prime}$ we obtained a dispersion of $1.8 \AA /$ pixel in the nominal range 3000-5300 A. Precisely, two 30-min spectra were obtained at mid-exposure time 02:13 UT of 9 August 2011.

Few additional 5500-8500 ^ spectra with dispersion $1.1 \AA /$ pix were moreover obtained on 23 and 28 June 2011 and on 1 and 7 July 2011 with the $0.6-\mathrm{m}$ and $0.7-\mathrm{m}$ reflector telescopes operated by the ANS Collaboration ${ }^{1}$ in La Polse di Cougnes (Udine, Italy) and at the Schiaparelli Observatory in Campo de' Fiori (Varese, Italy), respectively, to check for possible presence of $\mathrm{H}_{\alpha}$ emission, and confirming on all dates its absence.

The spectra, after correction for flat-field, bias and cosmicray rejection, were background subtracted and optimally extracted (Horne 1986) using IRAF ${ }^{2}$. Wavelength calibration was performed using comparison lamps acquired soon after each ontarget spectroscopic exposure, while flux calibration was accomplished by observing stars HR 8780 and HR 8634 from the Asiago internal list of bright spectrophotometric standard stars (Munari, in prep.). Wavelength calibration uncertainty was $\sim 0.5 \AA$; this was checked by using the positions of background night sky lines. The flux calibration of the Asiago spectra was verified against the source photometry acquired for this source (see below) and we found that it is correct to better than $5 \%$ across the optical continuum; the flux scale of the other spectra agrees with that of the Asiago ones. Spectra from a same observatory were then stacked together to increase the final signal-tonoise ratio.

Imaging data of the source were obtained with the robotic 14-inch Celestron telescope of the Sonoita Research Observatory (New Mexico, USA) in 2009 (from 15 October to 27 December) and 2010 (from 5 September to 17 November). The entire log of these observations is presented in Table 1. $B V R_{\mathrm{C}} I_{\mathrm{C}}$ Optec photometric filters were used; the detector was a $1024 \times 1024$ pixel SBIG STL-1001E CCD camera, with a $20^{\prime} \times$ $20^{\prime}$ field of view and a plate scale of $1^{\prime \prime} .25 \mathrm{pix}^{-1}$. The frames were corrected for bias and flat-field and reduced by means of simple aperture photometry. Photometric calibration was obtained through continous monitoring of several equatorial standards (Landolt 1992) during the nights of observations.

\subsection{X-rays}

The field of CGCS 5926 was observed on 6 January 2010 in the $0.3-10 \mathrm{keV}$ band with the X-ray Telescope (XRT; Burrows et al. 2005) on board the Swift satellite (Gehrels et al. 2005). The XRT pointing (ID: 00031572001) started at 08:34 UT, and $4374 \mathrm{~s}$ of on-source data were collected. The XRT data reduction was performed using the XRTDAs standard data pipeline package

\footnotetext{
${ }^{1}$ http://www.pd.astro.it/simbio-asiago/(in italian).

2 IRAF is the Image Analysis and Reduction Facility made available to the astronomical community by the National Optical Astronomy Observatories, which are operated by AURA, Inc., under contract with the US National Science Foundation. It is available at http://iraf. noao.edu/
} 
Table 1. Log and results of the optical $B V R_{\mathrm{C}} I_{\mathrm{C}}$ photometry of star CGCS 5926 presented in this paper (see text for details).

\begin{tabular}{|c|c|c|c|c|c|}
\hline HJD-2 450000 & V & $B-V$ & $V-R_{\mathrm{C}}$ & $R_{\mathrm{C}}-I_{\mathrm{C}}$ & $V-I_{\mathrm{C}}$ \\
\hline 5119.6871 & $14.949 \pm 0.007$ & $2.58 \pm 0.04$ & $1.789 \pm 0.008$ & $1.639 \pm 0.004$ & $3.432 \pm 0.007$ \\
\hline 5119.6978 & $14.978 \pm 0.007$ & $2.73 \pm 0.05$ & $1.819 \pm 0.008$ & $1.631 \pm 0.004$ & $3.453 \pm 0.007$ \\
\hline 5120.6184 & $14.948 \pm 0.008$ & $2.72 \pm 0.06$ & $1.852 \pm 0.009$ & $1.626 \pm 0.004$ & $3.479 \pm 0.008$ \\
\hline 5120.6294 & $14.935 \pm 0.007$ & $2.75 \pm 0.05$ & $1.824 \pm 0.008$ & $1.629 \pm 0.005$ & $3.458 \pm 0.008$ \\
\hline 5131.8875 & $14.881 \pm 0.008$ & $2.68 \pm 0.05$ & $1.794 \pm 0.009$ & $1.635 \pm 0.006$ & $3.432 \pm 0.010$ \\
\hline 5131.8974 & $14.916 \pm 0.008$ & $2.76 \pm 0.06$ & $1.827 \pm 0.009$ & $1.643 \pm 0.006$ & $3.475 \pm 0.009$ \\
\hline 5136.7797 & $14.881 \pm 0.012$ & $2.95 \pm 0.15$ & $1.800 \pm 0.013$ & $1.622 \pm 0.007$ & $3.427 \pm 0.014$ \\
\hline 5136.7897 & $14.869 \pm 0.012$ & $2.70 \pm 0.09$ & $1.798 \pm 0.013$ & $1.626 \pm 0.007$ & $3.424 \pm 0.014$ \\
\hline 5138.8442 & $14.863 \pm 0.013$ & $2.82 \pm 0.11$ & $1.794 \pm 0.014$ & $1.627 \pm 0.007$ & $3.424 \pm 0.014$ \\
\hline 5138.8542 & $14.876 \pm 0.013$ & $2.78 \pm 0.10$ & $1.835 \pm 0.014$ & $1.617 \pm 0.007$ & $3.453 \pm 0.014$ \\
\hline 5161.8343 & $14.688 \pm 0.009$ & $2.87 \pm 0.06$ & $1.745 \pm 0.012$ & $1.586 \pm 0.009$ & $3.328 \pm 0.011$ \\
\hline 5161.8542 & $14.665 \pm 0.012$ & $3.06 \pm 0.09$ & $1.757 \pm 0.014$ & $1.555 \pm 0.009$ & $3.305 \pm 0.014$ \\
\hline 5167.8041 & $14.693 \pm 0.016$ & $2.80 \pm 0.20$ & $1.751 \pm 0.016$ & $1.567 \pm 0.010$ & $3.312 \pm 0.018$ \\
\hline 5167.8223 & $14.684 \pm 0.015$ & $2.82 \pm 0.17$ & $1.750 \pm 0.016$ & $1.571 \pm 0.009$ & $3.318 \pm 0.016$ \\
\hline 5192.7497 & $14.844 \pm 0.015$ & $2.71 \pm 0.11$ & $1.750 \pm 0.013$ & $1.636 \pm 0.010$ & $3.389 \pm 0.016$ \\
\hline 5444.8529 & $14.824 \pm 0.006$ & $2.67 \pm 0.04$ & $1.798 \pm 0.007$ & $1.587 \pm 0.006$ & $3.370 \pm 0.007$ \\
\hline 5444.8675 & $14.814 \pm 0.006$ & $2.71 \pm 0.04$ & $1.775 \pm 0.007$ & $1.588 \pm 0.005$ & $3.353 \pm 0.008$ \\
\hline 5445.8451 & $14.819 \pm 0.006$ & $2.76 \pm 0.05$ & $1.774 \pm 0.007$ & $1.608 \pm 0.006$ & $3.372 \pm 0.008$ \\
\hline 5445.8597 & $14.824 \pm 0.006$ & $2.66 \pm 0.05$ & $1.781 \pm 0.007$ & $1.608 \pm 0.005$ & $3.379 \pm 0.007$ \\
\hline 5477.0016 & $14.752 \pm 0.010$ & $2.84 \pm 0.07$ & $1.772 \pm 0.009$ & $1.590 \pm 0.006$ & $3.353 \pm 0.010$ \\
\hline 5502.7452 & $14.809 \pm 0.008$ & $2.98 \pm 0.04$ & $1.770 \pm 0.008$ & $1.624 \pm 0.011$ & $3.398 \pm 0.014$ \\
\hline 5505.7462 & $14.820 \pm 0.008$ & $2.96 \pm 0.05$ & $1.785 \pm 0.010$ & $1.631 \pm 0.007$ & $3.421 \pm 0.009$ \\
\hline 5505.7650 & $14.812 \pm 0.010$ & $2.92 \pm 0.05$ & $1.778 \pm 0.012$ & $1.642 \pm 0.009$ & $3.403 \pm 0.014$ \\
\hline 5512.7498 & $14.841 \pm 0.007$ & $2.81 \pm 0.04$ & $1.782 \pm 0.009$ & $1.619 \pm 0.007$ & $3.407 \pm 0.009$ \\
\hline 5513.7223 & $14.833 \pm 0.007$ & $2.74 \pm 0.05$ & $1.775 \pm 0.010$ & $1.612 \pm 0.008$ & $3.393 \pm 0.009$ \\
\hline 5513.7413 & $14.833 \pm 0.009$ & $2.86 \pm 0.05$ & $1.780 \pm 0.011$ & $1.625 \pm 0.008$ & $3.413 \pm 0.012$ \\
\hline 5514.7398 & $14.838 \pm 0.007$ & $2.77 \pm 0.05$ & $1.783 \pm 0.010$ & $1.615 \pm 0.007$ & $3.403 \pm 0.009$ \\
\hline 5516.6971 & $14.810 \pm 0.008$ & $2.67 \pm 0.06$ & $1.753 \pm 0.010$ & $1.606 \pm 0.007$ & $3.366 \pm 0.012$ \\
\hline 5516.7161 & $14.820 \pm 0.008$ & $2.84 \pm 0.08$ & $1.760 \pm 0.010$ & $1.617 \pm 0.009$ & $3.386 \pm 0.010$ \\
\hline 5517.7169 & $14.812 \pm 0.009$ & $2.73 \pm 0.07$ & $1.769 \pm 0.009$ & $1.614 \pm 0.009$ & $3.395 \pm 0.010$ \\
\hline 5517.7369 & $14.842 \pm 0.008$ & $2.80 \pm 0.08$ & $1.792 \pm 0.011$ & $1.601 \pm 0.008$ & $3.395 \pm 0.011$ \\
\hline
\end{tabular}

(XRTPIPELINE V. 0.12.6), in order to produce screened event files. All data were extracted only in the photon counting (PC) mode (Hill et al. 2004), adopting the standard grade filtering (0-12 for PC) according to the XRT nomenclature, and using an extraction radius of $24^{\prime \prime}$.

Within the observation we analysed, with the tool XIMAGE V. 4.4.1, the $0.3-10 \mathrm{keV}$ image to search for sources detected (at a confidence level $>3 \sigma$ ) both at the optical position of star CGCS 5926 and inside the ROSAT error circle.

\subsection{Ultraviolet}

In parallel with the X-ray pointing, the UltraViolet-Optical Telescope (UVOT; Roming et al. 2005) onboard Swift as well, observed CGCS 5926 in the UVM2 band $(\lambda=2246 \AA$; full width at half maximum: $498 \AA$ ) for a total of 4364 s starting at 08:37:42 UT of 6 January 2010. Count rates were measured through aperture photometry using $5^{\prime \prime}$ apertures and were calibrated using the UVOT photometric system described by Poole et al. (2008).

\section{Results}

The optical spectrum of CGCS 5926 (Figs. 2 and 3) clearly shows the typical features of a carbon star (Yamashita 1967; Cohen et al. 1996): it is unmistakably dominated by the $\mathrm{C}_{2}$ Swan bands at 4737,5165 and $5635 \AA$ in the blue, and by $\mathrm{CN}$ bands redwards. We also find, among the main spectral features, the Na doublet at $5890 \AA$ and two atomic line blends of metal intersystem lines of Fe I, Ti I, Cr I, Ba I, Ca I, Mn I, Co I and Ni I located at $6352 \AA$ and $6497 \AA$ (see e.g. Turnshek et al. 1985). A telluric absorption feature is present at $7605 \AA$. No emission features typical of $\mathrm{X}$-ray binaries, such as Balmer and $\mathrm{He}$ II lines are present. In particular, no $\mathrm{H}_{\alpha}$ or $\mathrm{H}_{\beta}$ lines are readily detected either in emission or in absorption.

Moreover, the spectrum shown in Fig. 3 and covering the blue range of CGCS 5926 clearly indicates that there is no evident excess in the $U$ band, as no signal is detected from this object blueward of $\sim 4200 \AA$. This confirms the lack of signal detection in the blue part of the Asiago spectra acquired earlier, which is however not surprising given the red giant nature of the star coupled with the large optical absorption we infer toward it (see below).

Using the two-dimensional $\mathrm{C}(m, n)$ diagnostics of Yamashita (1967) and the quantitative classification scheme derived by Cohen (1979), we can better classify the spectral type of CGCS 5926. Concerning the $m$ parameter, which is associated with the temperature index $T$, we find from our optical spectrum of CGCS 5926 shown in Fig. 2 that $T=0.45 \pm 0.03$ : this, from Table 2 of Cohen (1979) implies that $m=6$, which corresponds to an effective blackbody temperature $T_{\text {eff }} \approx 2500 \mathrm{~K}$ (Cohen 1979; Bergeat et al. 2001). Likewise, the carbon indices $C 1$, $C 2$ and $C 3$ are $0.51 \pm 0.04,0.58 \pm 0.04$ and $0.90 \pm 0.06$, respectively, which allow us to infer that $n=2$ (see Table 3 of Cohen 1979). Therefore, we are able to classify the spectrum of CGCS 5926 as C $(6,2)$. We note that the use of the diagnostic $D$ of Cohen et al. (1996) instead of the temperature index $T$ for the determination of the parameter $m$ gives us a somewhat lower 


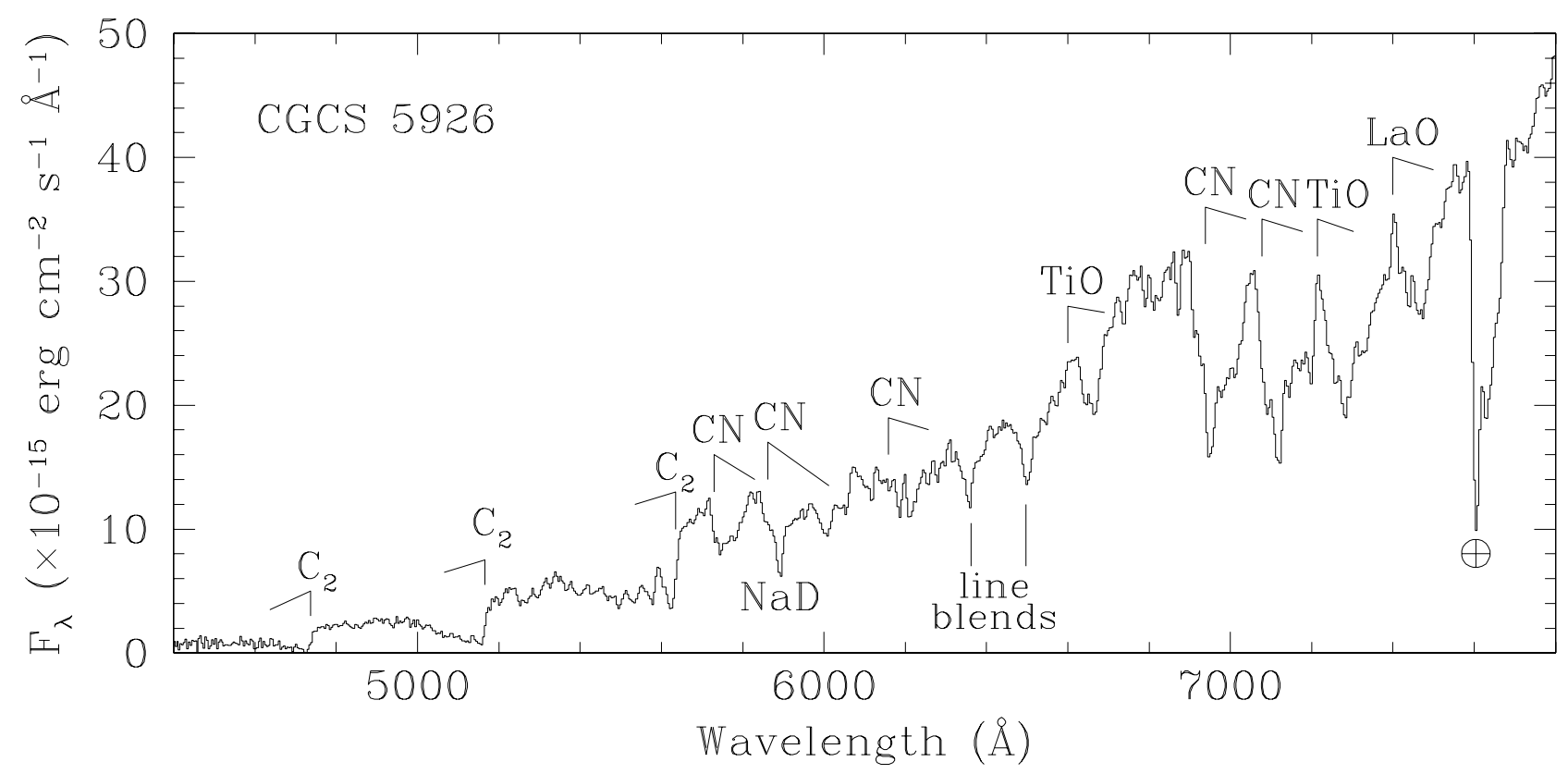

Fig. 2. 4400-7800 A optical spectrum of star CGCS 5926 obtained with the Copernicus telescope on 14 October 2009. The spectrum is typical of a late-type giant carbon star (see text). The telluric absorption band at $7600 \AA$ is marked with the symbol $\oplus$.

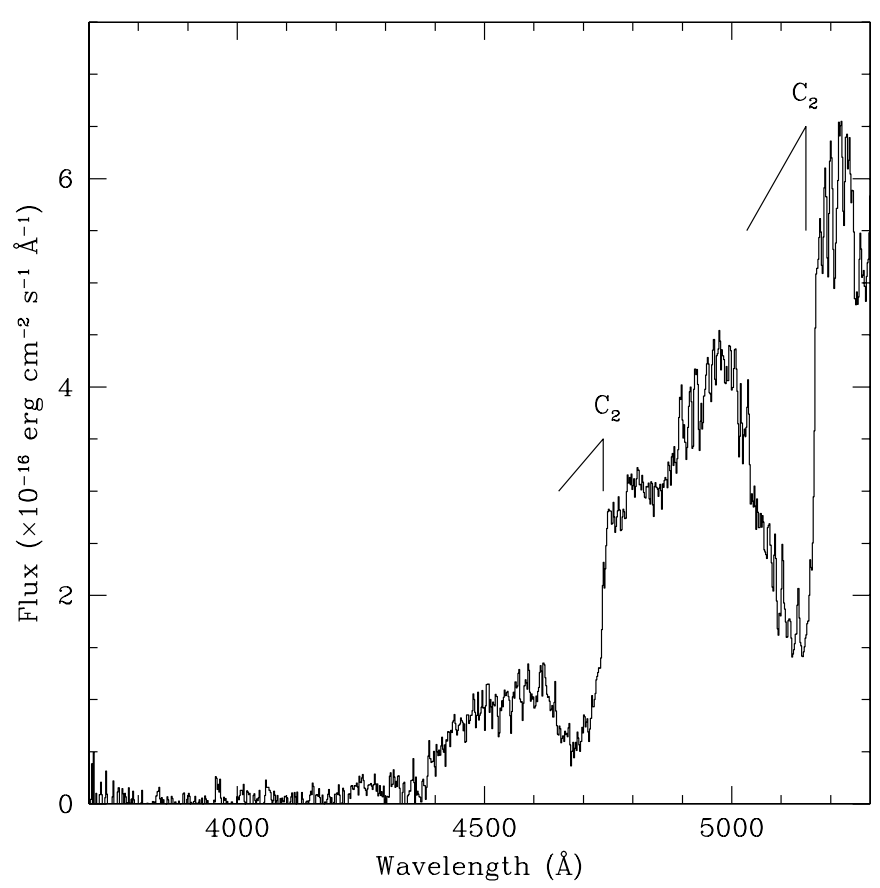

Fig. 3. 3700-5300 Å optical spectrum of star CGCS 5926 obtained with the Cassini telescope on 9 August 2011. The bluest $\mathrm{C}_{2}$ Swan bands are labeled. No signal is detected from the object blueward of $\sim 4200 \AA$.

value (around 5.5), implying a slightly higher temperature and thus an earlier spectral type.

The results of our photometry of CGCS 5926 are reported in Table 1 . These indicate that the source shows a variability of amplitude $\Delta m \sim 0.3 \mathrm{mag}$ on timescales of tens of days. Moreover, the $V-R_{\mathrm{C}}$ and $R_{\mathrm{C}}-I_{\mathrm{C}}$ color indices of CGCS 5926 seem to get smaller as the $V$-band magnitude decreases; that is, the star gets bluer with increasing brightness. Therefore we carried out a periodicity search on the photometric data using the Fourier code

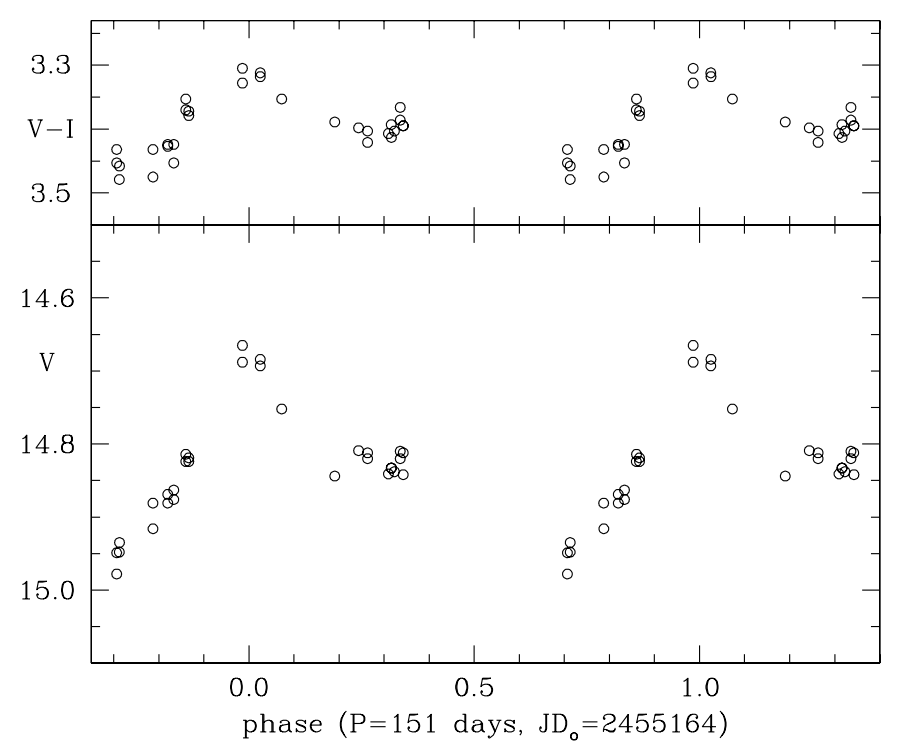

Fig. 4. $V$ (lower panel) and $V-I_{\mathrm{C}}$ (upper panel) light curves of CGCS 5926 folded with a period of 151 days and with zero phase at Julian Day 2455164 . We identify this as the radial pulsation periodicity of the star, which gets its bluest color at maximum brightness.

of Deeming (1975). A single, strong probability peak was found at a period of 151 days. The ephemeris we obtained with this period, expressed in Heliocentric Julian Days (HJDs), provides the following times of maxima in the $V$ band:

$T_{\max , V}=2455164( \pm 3)+(151 \pm 2) \times E$,

where $E$ is an integer number; the errors are at $3 \sigma$ confidence level. The $V$-band and the $V-I_{\mathrm{C}}$ color light curves of CGCS 5926 folded onto this ephemeris are presented in Fig. 4. Their shape, and the fact that the star gets bluer at maximum and redder at minimum optical brightness, suggest that this variability traces 
the radial pulsation of this carbon star (see e.g. Wallerstein \& Knapp 1998).

Because of the relatively short time baseline covered by our observations ( $\sim 400$ days, that is, a bit more than two pulsation cycles), we cannot build a mean light curve (i.e. one averaged over several and well covered pulsation cycles) for the star. Thus, the only feasible approach to estimate the above errors on the period and the epoch was to impose that the lightcurve is the simplest and smoothest possible, with a shape similar to that of other objects of that variability class (in this case, a rise to maximum faster than the decline to minimum). Besides, we stress that these stars usually display a significant variability of the period and epoch with time around mean values (possibly due to beating of multiple periodicities; see Wallerstein \& Knapp 1998, and references therein).

One can then estimate the distance to CGCS 5926 in the following manner. Assuming an average magnitude $V \sim 14.8$ for the source, its $J H K_{\mathrm{s}}$ magnitudes as reported in the 2MASS cata$\log$ (see Sect. 1) and the intrinsic color indices for carbon stars as reported in Table 6 of Ducati et al. (2001), one gets an average optical reddening $A_{V} \sim 3.8 \mathrm{mag}$, which corresponds to a color excess $E(B-V) \sim 1.23$ mag considering the Galactic absorption law of Cardelli et al. (1989) and a total-to-selective extinction ratio $R_{V}=3.1$ (Rieke \& Lebofski 1985). This implies that the intrinsic magnitude of the star is $V_{0} \sim 11.0$.

Therefore, given that a carbon star of spectral type $\mathrm{C}(6,2)$ has an absolute magnitude $M_{V} \approx-2.6$ (Cohen 1979), we obtain a distance $d \sim 5.2 \mathrm{kpc}$. This would locate CGCS 5926 in the far side of the outer part of the Perseus Arm of the Galaxy (see e.g. Fig. 1 of Leitch \& Vasisht 1998). The Galactic line-ofsight reddening in the direction of CGCS 5926 is $E(B-V)=$ 1.33 mag according to the maps of Schlegel et al. (1998). This value compares well with our reddening estimate for the source, thus indicating that this star is behind that Galactic Arm and that the observed reddening is likely due to interstellar absorption only, with no substantial contribution from material local to the source. We nevertheless note that the tabulated Galactic absorption maps should be treated with some degree of caution for objects which have the line of sight along the Galactic plane, such as the present case (for which the Galactic latitude is $\left.b=+0^{\circ} .96\right)$.

We remark that the above approach rules out the possibility that CGCS 5926 is a red supergiant of luminosity class I: indeed, in this case, its absolute magnitude would be $M_{V} \sim-5.6$ (Lang 1992), which would place the object at an uncomfortably large distance of $\sim 20 \mathrm{kpc}$, thus well outside the Galaxy given the Galactic longitude of the source $\left(l=115^{\circ} .5\right)$.

No X-ray source was detected in the XRT pointing, either at the optical position of CGCS 5926 or within the ROSAT error circle. Using the bayesian approach of Kraft et al. (1991), we get a 3- $\sigma$ confidence level upper limit count rate of $2 \times 10^{-3} \mathrm{~s}^{-1}$ in the $0.3-10 \mathrm{keV}$ energy band. This, assuming a Crab-like spectrum, corresponds to an observed flux limit of $\sim 9 \times$ $10^{-14} \mathrm{erg} \mathrm{cm}^{-2} \mathrm{~s}^{-1}$ in this energy range. Likewise, no UV source was detected in coincidence of the target down to a 3- $\sigma$ limit of 21.8 mag in the $U V M 2$ band.

The above Swift/XRT upper limit in X-rays can therefore be compared to the detection in the ROSAT faint source cata$\log$ : here the ROSAT source is reported with a $0.1-2.4 \mathrm{keV}$ count rate of $(1.96 \pm 0.76) \times 10^{-2} \mathrm{~s}^{-1}$. This, assuming again a Crablike spectrum, implies a flux of $\sim 1 \times 10^{-13} \mathrm{erg} \mathrm{cm}^{-2} \mathrm{~s}^{-1}$. It can be seen that the ROSAT spectral coverage is basically a subset of the XRT one; moreover, the contribution of the X-ray emission below $0.3 \mathrm{keV}$ to the total flux should not be relevant given the non-negligible line-of-sight absorption toward CGCS 5926 apparent from the optical data, which converts into a hydrogen column density $N_{\mathrm{H}} \sim 6.8 \times 10^{21} \mathrm{~cm}^{-2}$ if one uses the empirical formula of Predehl \& Schmitt (1995). The above figures thus suggest that, if the ROSAT detection is real, the source should possess variability at X-rays as well. We will discuss this in detail in the next section.

These fluxes, using the above distance estimate for CGCS 5926, thus imply an X-ray luminosity of $\approx 3 \times 10^{32} \mathrm{erg} \mathrm{s}^{-1}$ for the ROSAT measurement and an upper limit of comparable magnitude in the case of the Swift/XRT pointing.

To conclude this section, we note that the area of the sky containing CGCS 5926 was observed twice within the XMMNewton Slew Survey ${ }^{3}$ (Saxton et al. 2008) on 6 January 2007 and on 6 February 2009 for 7 and 9 s, respectively. For both observations, only a loose $3-\sigma$ upper limit of $\approx 2.5 \times 10^{-12} \mathrm{erg} \mathrm{cm}^{-2} \mathrm{~s}^{-1}$ in the $0.2-12 \mathrm{keV}$ band was obtained.

\section{Discussion}

Our optical spectroscopy of star CGCS 5926 confirms that this object can indeed be classified as a red giant carbon star; the optical photometry presented in this paper indicates that the source is slightly and periodically variable at least on timescales of a few months, which is also typical of this class of red giants and likely due to stellar pulsations (e.g. Wallerstein \& Knapp 1998). Our combination of optical and NIR photometry allowed us to determine a distance to the source $d \sim 5.2 \mathrm{kpc}$.

As shown in the previous section, no substantial continuum emission was detected in the blue part of the optical spectrum of CGCS 5926 (Fig. 3). The absence of a continuum excess of the order of few tenths of magnitude in the blue and in the ultraviolet, which is sometimes observed in SyXBs (see e.g. Gaudenzi \& Polcaro 1999), does not in itself rule out the classification of CGCS 5926 as a new member of this class of objects because of the large interstellar absorption we determine along the line of sight to this star. Indeed, our estimate implies about 6 mag of attenuation in the $U$ band assuming the law of Cardelli et al. (1989).

Similarly, the source was not detected in X-rays by Swift/XRT down to a limit of $3 \times 10^{32} \mathrm{erg} \mathrm{s}^{-1}$ in the $0.3-10 \mathrm{keV}$ band, which again may cast doubts on the possibility that this source can be a SyXB. Actually, the lowest fluxes detected in X-rays from this type of source (Masetti et al. 2002, 2007; Nespoli et al. 2010), are of the order of the above limit for CGCS 5926. Thus the X-ray nondetection does not in itself allow us to rule out that this star is the optical counterpart of a SyXB.

A possible explanation for the lack of an X-ray detection from the source is that we observed it during an eclipse of the compact accretor. Indeed a carbon star, being at the tip of the AGB, has a very large radius (e.g. Cohen 1979). Thus, when seen from the companion, it fills a large fraction of its sky, and as a matter of fact one third of all well studied symbiotic stars are eclipsing systems (Mikołajewska 2003). The geometric transit of the compact companion behind the cool giant can therefore take up to months, and the X-ray nondetection with Swift could be justified by the accreting component undergoing an eclipse at that time.

To explore this possibility we can first evaluate the size of the orbit in the case of accretion via the inner Lagrangian point.

\footnotetext{
3 See also

http://xmm.esac.esa.int/external/xmm_products/slew_ survey/upper_limit/uls.shtml
} 
In this occurrence, it is generally assumed that the size of the Roche lobe of a star in a binary system is characterized by its radius $R_{\mathrm{L}}$, defined as the radius of a sphere with the same volume of the Roche lobe. This quantity is related to the orbital semimajor axis $a$ according to the approximation of Eggleton (1983):

$$
\frac{R_{\mathrm{L}}}{a}=\frac{0.49 q^{2 / 3}}{0.6 q^{2 / 3}+\ln \left(1+q^{1 / 3}\right)},
$$

where $q$ is the mass ratio of the system. Assuming that the mass of the giant companion $M_{\mathrm{c}}$ is $\approx 1.4 M_{\odot}$ (we here use an average value; see Wallerstein \& Knapp 1998) and that the accretor is a NS (thus with a mass $M_{\mathrm{NS}} \approx 1.4 M_{\odot}$ ), we obtain that $q \approx 1$. Moreover, equating $R_{\mathrm{L}}$ to the typical radius of a $\mathrm{C}(6,2)$ giant carbon $\operatorname{star}\left(R \approx 470 R_{\odot}\right.$ : Cohen 1979), we get from Eq. (2) that $a \approx 1200 R_{\odot}$. This, by using Kepler's third law, implies an orbital period of $\approx 3000$ days and an inclination $i \gtrsim 70^{\circ}$ for the occurrence of an X-ray eclipse. This estimate, by the way, reinforces the fact that the periodicity we determine in the optical light curve is not of orbital nature but rather has its origin in the pulsation of the stellar structure of CGCS 5926.

However, in the present case the matter transfer onto the compact object is unlikely to be occurring via Roche lobe overflow as this would produce X-ray emission which is $\sim 5$ orders of magnitude more intense than the upper limit that we obtained with Swift/XRT (as for example in GX 1+4: Chakrabarty $\&$ Roche 1997); our very detection of regular radial pulsations reinforces this assumption. Therefore, the above figures should rather be considered as hard lower limits for orbital period and inclination.

The mass transfer would thus take place via a stellar wind, which makes the overall accretion mechanism much less efficient and the X-ray luminosity much lower (but see Mohamed \& Podsiadlowski 2007, 2011): in this case the orbit will be much wider.

Assuming thus the orbital period to be larger by at least a factor of 4 with respect to the above estimate, and again using Kepler's third law, we get $a \gtrsim 3100 R_{\odot}$ : this means that, in order to observe an eclipse from such a system, its inclination should be $\gtrsim 80^{\circ}$. In this case, the eclipse would last not more than $\sim 75$ days, which is a tiny fraction (less than $1 \%$ ) of the orbital period. This however tells us that, even if with admittedly a low level of probability, the hypothesis of an eclipsing SyXB cannot be completely discarded.

A less contrived scenario is possibly the one in which we consider that the source is highly variable in X-rays, as seen in other SyXBs. For instance, the X-ray fluxes of 4U 1700+24 (Masetti et al. 2002) and 4U 1954+31 (Masetti et al. 2006b) span 2 orders of magnitude; likewise, and in a more extreme manner, that of IGR J16358-4726 spans a dynamical range of $10^{4}$ (Patel et al. 2007; Nespoli et al. 2010). This can be qualitatively explained by accretion from an inhomogeneous stellar wind (possibly modulated by stellar pulsations) coming from a red giant star, possibly coupled with an elliptical orbit of the accretor.

If we assume that the X-ray luminosity coming from CGCS 5926 and detected with ROSAT is due to accretion from a red giant stellar wind, an accretion rate onto the compact star of $\dot{M} \approx 3 \times 10^{-14} M_{\odot} \mathrm{yr}^{-1}$ is inferred. This can be interpreted by assuming a typical mass loss rate via stellar wind of a giant carbon star $\left(\dot{M} \approx 10^{-7} M_{\odot} \mathrm{yr}^{-1}\right.$, and sometimes higher; e.g. Wallerstein $\&$ Knapp 1998) and an accretion efficiency $\eta=10^{-4}$ which is quite normal for a compact object accreting from a stellar wind (e.g. Frank et al. 1992). In the case that the accreting object is a NS, the remaining difference between the above two values of $\dot{M}$ can possibly be accounted for by a partial inhibition of the accretion due to the "propeller effect" (Illarionov \& Sunyaev 1975), according to which the magnetosphere of the NS acts as a barrier to accretion of matter onto the NS surface. It is however noted that this star is not detected in the IRAS far-infrared (FIR) source catalogue (IRAS 1986). Given the direct correlation between the mass loss in carbon stars and their FIR emission (e.g. Wallerstein \& Knapp 1998), we may expect that the $\dot{M}$ of CGCS 5926 is actually substantially lower than $10^{-7} M_{\odot} \mathrm{yr}^{-1}$. This would make the propeller effect hypothesis more viable to explain the low level of X-ray emission from CGCS 5296 assuming that this star is part of a SyXB system.

It is prudent to verify whether the X-ray emission detected with ROSAT is indeed associated with CGCS 5926 rather than, for instance, other objects within the X-ray error circle (see Fig. 1). In order to check this, we here evaluate the probability of finding a late-type giant star in a $20^{\prime \prime}$-radius circle at these Galactic latitudes. To this aim, we first made extensive catalogue searches using the SIMBAD and VIZIER databases (see for instance Maehara \& Soyano 1999, and references therein). We found that the total number of known Carbon stars in our Galaxy is less than 2000 (including suspected and tentative ones). Even assuming the extreme case in which they are all concentrated in a strip on the Milky Way plane with Galactic latitude between $b=-5^{\circ}$ and $b=+5^{\circ}$, we get a density of $\lesssim 0.5$ carbon stars per square degree; this in turn implies a probability less than $5 \times 10^{-5}$ to have one such star in a circle of radius $20^{\prime \prime}$.

A more conservative estimate can be obtained using the "Besançon" Galactic model (Robin et al. 2003). This population synthesis description of the Galaxy returned a total probability of $5.0 \times 10^{-3}$ that, within a circle of radius $20^{\prime \prime}$ around the position of the ROSAT source 1RXS J234545.9+625256, we find an M-type red giant of luminosity class I, II or III. Thus, the probability of finding a red giant star within the ROSAT error circle at the Galactic coordinates of CGCS 5926 is a number between the two estimates above, and thus of the order of $\approx 10^{-4}$. This suggests that the chance probability of the positional coincidence between the star CGCS 5926 and the ROSAT source 1RXS J234545.9+625256 is quite low, although not vanishing. We thus remain with an admittedly very small possibility that the two objects are actually not correlated.

One may thus wonder whether other classes of Galactic or extragalactic X-ray sources may display this variable highenergy behaviour, so that one of the other fainter optical objects within the ROSAT error circle in Fig. 1 may be the actual counterpart of the X-ray emitter. For instance, the ROSAT detection may have been produced because of magnetic activity from Galactic objects such as a "flare star" (e.g. Pettersen 1989), or by the outburst of a very faint X-ray transient (VFXT) LMXB (see e.g. Degenaar \& Wijnands 2009).

The latter objects are however mostly associated with old (bulge) stellar population, so we do not expect to find one of them far from the inner parts of the Galaxy. Moreover, and most importantly, these transients reach X-ray luminosities of at least $10^{34} \mathrm{erg} \mathrm{s}^{-1}$ (e.g., Degenaar \& Wijnands 2009), which means that the ROSAT source would lie at a distance of at least $29 \mathrm{kpc}$, that is, well outside the Galaxy. Therefore, the VFXT hypothesis is not tenable for the ROSAT detection.

The possibility that one of the other sources within the ROSAT error circle (see Fig. 1) is a dwarf, late-type flare star of UV Cet type cannot of course be excluded a priori, especially given that we have no specific information on any of them. 
However, using the USNO-A2.04 magnitudes of these sources we find that the brighter ones are too blue to be M-type dwarfs, while the fainter ones lie at distances $d \gtrsim 700 \mathrm{pc}$ (assuming an absolute magnitude $M_{R} \approx+10$; e.g., Lang 1992); this would mean that the ROSAT detection implies an X-ray luminosity $z 3 \times 10^{30} \mathrm{erg} \mathrm{s}^{-1}$, which is extreme for an eruption of a flare star (see for instance Pandey \& Singh 2008). We caution the reader that the USNO-A2.0 data can have systematic uncertainties of a few tenths of magnitude (see Masetti et al. 2003), and that in the above considerations we did not take into account the interstellar reddening (which would move the above upper limit on the distance closer to earth and would relax the X-ray luminosity constraint). However, we do not think that the latter is a real issue because we expect that the bulk of the absorption lies within the Perseus arm, that is, at a distance larger than $\sim 2 \mathrm{kpc}$ from earth according to Fig. 1 of Leitch \& Vasisht (1998). Thus, although more reasonable than the VFXT one, we deem the flare star interpretation for the ROSAT detection still unlikely.

We can in any case definitely exclude a flare star nature for CGCS 5926 given that it is an evolved star, whereas those are typically young and fast rotating stellar objects; moreover, these objects have X-ray luminosities which rarely exceed $10^{30} \mathrm{erg} \mathrm{s}^{-1}$ (e.g., Pandey \& Singh 2008), which means 2 orders of magnitude less that the ROSAT detection in case it was emitted by CGCS 5926. Similarly, this red star cannot be associated with a very faint X-ray transient as these systems are in general "normal" LMXBs with a late-type (or sometimes a degenerate) dwarf as donor star.

As regards the possibility of an extragalactic object being responsible for the X-ray emission detected with ROSAT, we consider that the most likely case would be that of a background active galactic nucleus (AGN). In order to evaluate the probability of the occurrence of such a source within the ROSAT error circle we use the AGN density relation of Cappelluti et al. (2007). We see that the chance of finding a field object with a ROSAT X-ray flux larger than $\approx 10^{-13} \mathrm{erg} \mathrm{cm}^{-2} \mathrm{~s}^{-1}$ in a $20^{\prime \prime}$ radius circle is $\approx 2 \times 10^{-4}$ : this again implies a very low, although not completely negligible, chance coincidence probability that the ROSAT detection was due to an AGN located beyond the Galactic plane.

Finally, the only alternative explanation that may come to mind is that the low confidence level detection with ROSAT is actually spurious, and that CGCS 5926 does not emit appreciable X-rays, especially above $2 \mathrm{keV}$. This may suggest that no accretion onto a compact companion is taking place. If this interpretation is correct, CGCS 5926 can be discarded as a further possible SyXB. Moreover, as our data do not give us any hint about this, again there may possibly be no companion at all for CGCS 5926, making it a single, isolated carbon star. In this case, we remark that our X-ray flux limit would still be compatible with the coronal emission from a late-type giant star, this being generally about 3 orders of magnitude lower than our result (Hünsch et al. 1998).

\section{Conclusions}

We performed a multiwavelength study of star CGCS 5926 in X-ray, ultraviolet, optical and NIR bands using new observations as well as archival data when available. This was done in order to investigate the possible SyXB nature of this source

\footnotetext{
4 Available at

http://archive. eso.org/skycat/servers/usnoa/
}

due to its positional proximity with the ROSAT X-ray source 1RXS J234545.9+625256.

The optical data confirm that the object is a carbon star likely located at $\sim 5.2 \mathrm{kpc}$ from earth and show the presence of radial pulsations with a periodicity of 151 days. No detection of X-ray or ultraviolet emission was obtained from the star in 2010, nor was any excess detected in the blue part of its optical spectrum. While all of this casts doubts on the former ROSAT detection and even on the possibility that this source is a SyXB (or even a binary system at all), our multiwavelength data analysis does not rule out either of these possibilities. Alternative interpretations for the variable X-ray emission, such as an interloping flare star, a field VFXT or a background AGN were found to be highly unlikely or were definitely ruled out. Thus, we remain with the two possibilities above: either CGCS 5926 is indeed a SyXB, or the ROSAT source is spurious.

Future spectrophotometric optical and NIR monitoring over a long time baseline (years) will help determine if indeed CGCS 5926 is part of a binary system, while deeper, longer, and higher sensitivity X-ray observations with satellites such as XMM-Newton or Chandra will provide a possible detection of, or at least tighter upper limits on, the high-energy emission from this source.

Acknowledgements. We are grateful to the following people for their assistance in the acquisition of the optical spectra of CGCS 5926: Alessandro Siviero (Asiago), Gianni Cetrulo and Antonio Englaro (La Polse di Cougnes), Alberto Milani (Campo de' Fiori), and Silvia Galleti and Roberto Gualandi (Loiano). We also thank Mauro Dadina and Isabella Pagano for discussions, and the anonymous referee for useful remarks which helped us to improve the quality of this paper. This research has made use of the ASI Science Data Center Multimission Archive; it also used the NASA Astrophysics Data System Abstract Service and the NASA/IPAC Infrared Science Archive, which are operated by the Jet Propulsion Laboratory, California Institute of Technology, under contract with the National Aeronautics and Space Administration. This publication made use of data products from the Two Micron All Sky Survey (2MASS), which is a joint project of the University of Massachusetts and the Infrared Processing and Analysis Center/California Institute of Technology, funded by the National Aeronautics and Space Administration and the National Science Foundation. This research has also made extensive use of the SIMBAD and VIZIER databases operated at CDS, Strasbourg, France. N.M. acknowledges financial contribution from the ASI-INAF agreement No. I/009/10/0. K.L.P. and J.P.O. acknowledge the support of the UK Space Agency. S.S. acknowledges partial support from NSF and NASA grants to ASU.

\section{References}

Bergeat, J., Knapik, A., \& Rutily, B. 2001, A\&A, 369, 178

Burrows, D. N., Hill, J. E., Nousek, J. A., et al. 2005, Space Sci. Rev., 120, 165 Cappelluti, N., Hasinger, G., Brusa, M., et al. 2007, ApJS, 172, 341 Cardelli, J. A., Clayton, G. C., \& Mathis, J. S. 1989, ApJ, 345, 245

Chakrabarty, D., \& Roche, P. 1997, ApJ, 489, 254

Cohen, M. 1979, MNRAS, 186, 837

Cohen, M., Wainscoat, R. J., Walker, H. J., \& Volk, K. 1996, AJ, 111, 1333

Corbet, R. H. D., Sokoloski, J. L., Mukai, K., Markwardt, C. B., \& Tueller, J. 2008, ApJ, 675, 1424

Corbet, R. H. D., Krimm, H. A., Barthelmy, S. D., et al. 2010, ATel, 2570

Deeming, T. J. 1975, Ap. Space Sci., 36, 137

Degenaar, N., \& Wijnands, R. 2009, A\&A, 495, 547

Ducati, J. R., Bevilacqua, C. M., Rembold, S. B., \& Ribeiro, D. 2001, ApJ, 558, 309

Eggleton, P. P. 1983, ApJ, 268, 368

Farrell, S. A., Gosling, A. J., Webb, N. A., et al. 2010, A\&A, 523, A50

Frank, J., King, A. R., \& Raine, D. J. 1992, Accretion Power in Astrophysics (Cambridge: Cambridge Univ. Press)

Gaudenzi, S., \& Polcaro, V. F. 1999, A\&A, 347, 473

Gehrels, N., Chincarini, G., Giommi, P., et al. 2004, ApJ, 611, 1005

Hill, J. E., Burrows, D. N., Nousek, J. A., et al. 2004, Proc. SPIE, 5165, 217

Hinkle, K. H., Fekel, F. C., Joyce, R. R., et al. 2006, ApJ, 641, 479

Horne, K. 1986, PASP, 98, 609

Hünsch, M., Schmitt, J. H. M. M., Schröder, K.-P., \& Zickgraf, F.-J. 1998, A\&A, 330,225 
Illarionov, A. F., \& Sunyaev, R. A. 1975, A\&A, 39, 185

IRAS 1986, IRAS Catalog of Point Sources, Version 2.0

Kraft, R. P., Burrows, D. N., \& Nousek, J. A. 1991, ApJ, 374, 344

Landolt, A. U. 1992, AJ, 104, 340

Lang, K. R. 1992, Astrophysical Data: Planets and Stars (New York: Springer-Verlag)

Leitch, E. M., \& Vasisht, G. 1998, New Astron., 3, 51

Liu, Q. Z., van Paradijs, J., \& van den Heuvel, E. P. J. 2007, A\&A, 469, 807

Maehara, H., \& Soyano, T. 1987, Ann. Tokyo Astron. Obs., 21, 293

Maehara, H., \& Soyano, T. 1999, Publ. Nat. Astron. Obs. Japan, 5, 149

Masetti, N., Dal Fiume, D., Cusumano, G., et al. 2002, A\&A, 382, 104

Masetti, N., Palazzi, E., Pian, E., et al. 2003, A\&A, 404, 465

Masetti, N., Orlandini, M., Palazzi, E., Amati, L., \& Frontera, F. 2006a, A\&A, 453, 295

Masetti, N., Rigon, E., Maiorano, E., et al. 2006b, A\&A, 464, 277

Masetti, N., Landi, R., Pretorius, M. L., et al. 2007, A\&A, 470, 331

Mikołajewska, J. 2003, Orbital and Stellar Parameters of Symbiotic Stars, in Symbiotic Stars Probing Stellar Evolution, ed. R. L. M. Corradi, J. Mikołajewska, \& T. J. Mahoney, ASP Conf. Ser. (San Francisco: ASP), 303, 9

Mohamed, S., \& Podsiadlowski, P. 2007, Wind Roche-Lobe Overflow: a New Mass-Transfer Mode for Wide Binaries, in 15th European Workshop on White Dwarfs, ed. R. Napiwotzki, \& M. R. Burleigh (San Francisco: ASP), ASP Conf. Ser., 372, 397
Mohamed, S., \& Podsiadlowski, P. 2011, in Asiago Meeting on Symbiotic Stars, ed. A. Siviero, \& U. Munari, Balt. Astron. Spec. Issue, in press

Munari, U., \& Zwitter, T. 2002, A\&A, 383, 188

Nespoli, E., Fabregat, J., \& Mennickent, R. E. 2010, A\&A, 516, A94

Nucita, A. A., Carpano, S., \& Guainazzi, M. 2007, A\&A, 474, L1

Pandey, J. C., \& Singh, K. P. 2008, MNRAS, 387, 1627

Patel, S. K., Zurita, J., Del Santo, M., et al. 2007, ApJ, 657, 994

Pettersen, B. R. 1989, Sol. Phys., 121, 299

Poole, T. S., Breeveld, A. A., Page, M. J., et al. 2008, MNRAS, 383, 627

Predehl, P., \& Schmitt, J. H. M. M. 1995, A\&A, 293, 889

Rieke, G. H., \& Lebofsky, M. J. 1985, ApJ, 288, 618

Robin, A. C., Reylé, C., Derriére, S., \& Picaud, S. 2003, A\&A, 409, 523

Roming, P. W. A., Kennedy, T. E., Mason, K. O., et al. 2005, Space Sci. Rev., 120,95

Saxton, R. D., Read, A. M., Esquej, P., et al. 2008, A\&A, 480, 611

Schlegel, D. J., Finkbeiner, D. P., \& Davis, M. 1998, ApJ, 500, 525

Skrutskie, M. F., Cutri, R. M., Stiening, R., et al. 2006, AJ, 131, 1163

Thompson, T. W. J., Tomsick, J. A., Rothschild, R. E., in 't Zand, J. J. M., \& Walter, R. 2006, ApJ, 649, 373

Turnshek, D. E., Turnshek, D. A., Craine, E. C., \& Boeshaar, P. C. 1985, An atlas of digital spectra of cool stars, Western Research Company, Tucson

Voges, W., Aschenbach, B., Boller, T., et al. 2000, IAU Circ., 7432

Wallerstein, G., \& Knapp, G. R. 1998, ARA\&A, 36, 369

Yamashita, Y. 1967, Publ. DAO, 13, 67 